\title{
Optimal Node Placement in Underwater Wireless Sensor Networks
}

\author{
Muhamad Felemban*, Basem Shihada ${ }^{\dagger}$, Kamran Jamshaid $^{\dagger}$ \\ * Department of Computer Science, Purdue University, USA, mfelemba@purdue.edu \\ $\dagger$ CEMSE Division, KAUST, Saudi Arabia, \{basem.shihada, kamran.jamshaid\}@kaust.edu.sa
}

\begin{abstract}
Wireless Sensor Networks (WSN) are expected to play a vital role in the exploration and monitoring of underwater areas which are not easily reachable by humans. However, underwater communication via acoustic waves is subject to several performance limitations that are very different from those used for terresstrial networks. In this paper, we investigate node placement for building an initial underwater WSN infrastructure. We formulate this problem as a nonlinear mathematical program with the objective of minimizing the total transmission loss under a given number of sensor nodes and targeted coverage volume. The obtained solution is the location of each node represented via a truncated octahedron to fill out the 3D space. Experiments are conducted to verify the proposed formulation, which is solved using Matlab optimization tool. Simulation is also conducted using an ns-3 simulator, and the simulation results are consistent with the obtained results from mathematical model with less than 10 error.
\end{abstract}

Keywords-UWSN, underwater node deployment, optimization

\section{INTRODUCTION}

Water covers almost $70 \%$ of the planet Earth. Most of this underwater environment is still unexplored. There is an increasing interest in exploring and monitoring this environment. Autonomous Underwater Vehicles (AUVs) have been used in recent years to monitor and collect data from the ocean floor. AUVs can be operated without tethers or cables. However, underwater communication challenges limit the performance of these AUVs. These challenges include limited bandwidth of the underwater communication channels, high path loss and multi-path fading, large propagation delays, and high bit error rate (BER). These challenges can be addressed by introducing large-scale wireless sensor nodes that transmit the control data over multi-hop routes wirelessly from the surface to the AUVs, while also relaying the data sensed by the AUVs back to the surface.

Underwater Wireless Sensor Network (UWSN) is a promising research field. UWSN have been extensively used in many submarine applications for data sampling, environmental monitoring, disaster prevention, and other military applications. UWSN offers many advantages over the traditional underwater sensing techniques [8], including real-time data monitoring, online system configuration, instant failure detection, and unlimited storage capacity. Nodes in a vast UWSN that cover a sparse area need to be optimally placed to reduce per node energy consumption. As the distance between the nodes increases, more energy is required to maintain a reasonable Signal-to-Noise (SNR) value over longer distances.
Thus, nodes placement is a fundamental research challenge in building a UWSN.

In this paper, we focus on finding the optimal positioning strategy for sensor nodes which support AUV's missioncritical operation. The deployment problem is formulated as an optimization problem as follows: There exists a volume of interest $V$ with a single or multiple AUVs operating on a mission-critical task. UWSN is required to relay control packets from the surface nodes to AUVs and the data packets from AUVs to surface nodes. Our main objective is to define a placement strategy for nodes such that it attains maximum coverage and connectivity with minimum transmission loss. The inter-node distance depends on the transmission loss at given depth. The optimum inter-node distance is found by finding the optimal transmission loss between the nodes. Once the inter-node distance is determined, we also obtain the minimum number of nodes that covers a certain volume and the maximum coverage volume for a given number of nodes.

The rest of this paper is organized as follows. Sect. II provides some previous work in UWSN deployment. Sect. III presents the network model and the problem formulation. Sect. IV discusses the analytic results. In Sect. V we present the simulation setup and performance results. We conclude by summarizing our contributions and identifying directions for future research in Sect. VI.

\section{RELATED WORK}

A number of techniques have been proposed for optimal deployment of UWSN to achieve full coverage and connectivity. In [3], a distributed node deployment technique for UWSN is presented. The proposed solution is an iterative algorithm that adjusts the node's depth after the initial deployment until no further improvement can be done. The algorithm achieves maximum network connectivity and sensing coverage with the available number of nodes. Unlike our work, the nodes are first deployed randomly at the bottom of the water and then adjust their depths based on the respective distances from neighbors. In our paper, the position of each node is determined prior to the deployment. In [9], [14], different deployment strategies for surface gateways are presented. The problem is modeled as a 3D graph optimization problem with objective function of finding the subset of candidate positions for the gateway nodes to satisfy certain constraints. However, the goal in this paper is to find the best locations of the nodes inside the water given that the location of the gateways nodes are known. 
Different node deployment strategies for 2D and 3D architecture for underwater sensor network have been been studied in [4]. Their objective is to determine the minimum number of nodes that achieves optimal sensing and coverage range. Similarly, [5] proposed nodes placement strategy that achieves full sensing coverage in 3D space using the minimum number of nodes. The strategy is based on Voronoi tessellation of certain polyhedrons in the 3D space. A metric called volumetric quotient is introduced to measure the ratio of the volume of the polyhedron to the volume of the node's sensing range cicrumsphere of radius R. High volumetric quotient means that the polyhedron is completely space-filling the cicrumsphere of radius $\mathrm{R}$ of the node and hence less number of nodes is required to cover the $3 \mathrm{D}$ space. The problem is reformulated as finding the best polyhedron that gains the highest volumetric quotient given the radius R. In both papers, the nodes locations are found geometrically and independently of acoustic communication characteristics. Our work, on the other hand, reflects the effects of transmission loss on the deployment of the sensor nodes.

The work in this paper addresses similar problem as in [10], where the problem of placing wireless nodes in shadow zones is tackled. The optimal placement of affected nodes is found using a mathematical model to minimize the transmission loss while maintaining the network connectivity. The problem of finding the new location of the introduced node is formulated as a nonlinear programming problem. The objective function is to find the optimal location that minimizes the transmission loss between the two nodes. However, the objective function in this paper is to minimize the transmission loss under given number of sensor nodes and targeted volume. Our work incorporates the strategy proposed in [5] to find the nodes location respectively.

\section{Methodology}

\section{A. Network Model}

We consider a 3D UWSN where a certain number of nodes are deployed to cover certain underwater volume. The underwater network consists of two entities: Surface Gateways (SG) and Relay Nodes (RN). SGs are static nodes attached to buoys on the surface. They are equipped with two types of interfaces: acoustic and electromagnetic. SGs connect the underwater network to the Internet via electromagnetic interface. SG forwards and receives packets to the underwater network using acoustic interface. Each SG can be connected to one or more RNs. RNs are placed at multiple depths inside the water to relay the packets from SGs to the operating AUVs at the ocean floor and vice versa. In our network model, we assume:

- All RNs are equipped with homogenous transceivers and have a sphere-based communication with a radius of $r . r$ is assumed to be constant for all nodes. We further assume that all nodes in the network transmit with a uniform transmission power

- Two nodes are connected if the node inter-distance is less than or equal $r$

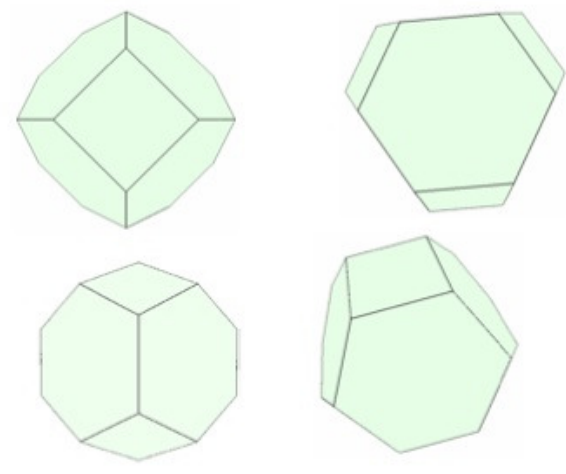

Fig. 1. Truncated Octahedron [5]

- The network is fairly large and there is no boundary effect. The number of RNs is inversely proportional to the volume covered by the RNs

- The ocean is divided horizontally into different regions based on the depth. The propagation characteristics of acoustic waves are different in each region

- Each RN can be deployed in any position at any depth. Furthermore, RNs maintain their location using various means of location and depth adjustments

The goal is to find an optimal placement strategy that achieves full coverage and full connectivity with all direct neighboring RNs with minimum transmission loss. Full coverage and connectivity can be achieved by utilizing the solution suggested in [5]. The solution starts with finding a space-filling polyhedron that best approximates the sensing sphere. This is measured by the volumetric quotient, which measures the ratio of the volume of the polyhedron to the volume of the communication sphere of radius $r$. It is found that Truncated Octahedron (TO) has the highest volumetric quotient among all other polyhedrons. A TO has 14 faces, 8 of which are hexagonal and 6 are square as shown in Figure 1. The length of the edge in the hexagonal and square face is $a$. The volume of a TO is $8 \sqrt{2} a^{3}$ and the radius of its circumsphere is $\frac{\sqrt{10 a}}{2}$. The volumetric quotient of TO is

$$
8 \sqrt{2} a^{3} / \frac{4}{3} \pi\left(\frac{1}{2} \sqrt{10} a\right)^{3}=24 / 5 \sqrt{5} \pi=0.68329
$$

The placement algorithm then finds the locations where RNs should be placed to tessellate the space-filling polyhedron, i.e., TOs. The input of the algorithm is the radius of the circumsphere of TO $R$ and the co-ordinates of a seed point, e.g., $(x, y, z)$. The output is the coordinates of the locations where RNs are to be placed. The coordinates of the RNs locations with an arbitrary seed-point $(c x, c y, c z)$ are as follows:

$$
\left(c x+(2 u+w) \frac{2 R}{\sqrt{5}}, c y+(2 v+w) \frac{2 R}{\sqrt{5}}, c z+w \frac{2 R}{\sqrt{5}}\right)
$$

where $u \in Z, v \in Z, w \in Z ; Z$ is the set of positive and negative integers. To achieve full coverage and connectivity of the network, $R$ is set to $r$. Since the distance between any two nodes should be less than or equal $r$, we reduce the problem 
to be finding the optimal value of $r$ that minimizes the number of nodes and the transmission loss.

\section{B. Acoustic Communication Characteristics}

The SNR of an underwater acoustic signal at a receiver can be expressed in $\mathrm{dB}$ by the passive sonar equation [6]

$$
S N R=S L-T L-N L+D I
$$

where $S L$ is the signal level of transmission power which is related to the transmission power intensity $I_{t}$ and hence to the transmission power $P_{t}$ of the transceiver. The intensity $I_{t}$ in shallow water is given in $W a t t s / m^{2}$ as follows,

$$
I_{t}=\frac{P_{t}}{2 \pi \times 1 m \times z}
$$

where $z$ is the depth in meters. In deep water Eq. (3) becomes,

$$
I_{t}=\frac{P_{t}}{4 \pi \times 1 m \times z}
$$

and $S L$ is given as,

$$
S L=10 \log \left(\frac{I_{t}}{0.67 \times 10^{-18}}\right)
$$

$D I$ is the directivity index and is set to zero and $N L$ is the ambient noise level in ocean and is given by Eq. (6). The noise is limited to four common sources: water turbulence $N_{t}$, surface-ship $N_{s}$, thermal noise $N_{t h}$, and breaking waves $N_{w}$. Those sources can be described using Gaussian statistics and power spectral density (PSD) in $\mathrm{dB}$ re $\mu$ per $\mathrm{Hz}$ as follows [7],

$$
N(f)=N_{t}(f)+N_{s}(f)+N_{t h}(f)+N_{w}(f)
$$

$T L$ is the transmission loss in acoustic waves which is caused by two phenomena: energy spreading and waves absorption. Energy spreading, also called geometric spreading, mainly depends on the transmission range of the acoustic waves [6]. Waves absorption, on the other hand, is frequency-dependent. High-frequency signals are more vulnerable to absorption losses because of the transference of the acoustic energy to heat. The transmission loss, $\delta$, of acoustic signal in $\mathrm{dB}$ with transmission range $r$, in meters, and frequency $f$, in $\mathrm{KHz}$, is given by [1],

$$
\delta=\kappa \log (r)+\alpha(f) r 10^{-3}
$$

where $\alpha(f)$ is the absorption coefficient and $\kappa$ is the spreading factor caused by the energy spreading. Commonly used values for $\kappa=10$ for spherical spreading, $\kappa=20$ for cylindrical spreading, and $\kappa=15$ for practical spreading. The absorption coefficient of the seawater can be expressed using different models. A simple yet an accurate absorption model is presented by Ainslie and McColm [11], [13] as follows:

$$
\alpha(f)=\gamma_{1} \frac{f_{1} f^{2}}{f_{1}+f^{2}}+\gamma_{2} \frac{f_{2} f^{2}}{f_{2}+f^{2}}+\gamma_{3} f^{2}
$$

where,

$$
\begin{aligned}
f_{1} & =0.78(S / 35)^{\frac{1}{2}} e^{\frac{T}{26}} \\
f_{2} & =42 e^{\frac{T}{17}} \\
\gamma_{1} & =0.106 e^{\frac{P H-8}{0.56}} \\
\gamma_{2} & =0.52\left(1+\frac{T}{43}\right)\left(\frac{S}{35}\right) e^{\frac{-d}{6}} \\
\gamma_{3} & =0.00049 e^{-\left(\frac{T}{27}+\frac{d}{17}\right)}
\end{aligned}
$$

where $T$ is the temperature in $C^{\circ}, d$ is the depth in $m, p H$ is water acidity, and $S$ is the water salinity. The default values of $p H$ and $S$ are 8 and 35, respectively.

\section{Problem Formulation}

We formulate the optimization problem as follows. Let the network consist of $\mathrm{N}$ number of RN's with the same acoustic propagation characteristics at a given depth $d$ and temperature $T$. The goal of the optimization problem is to find the optimal distance between nodes, i.e., $r$, for a fixed value of $f$ that covers a volume $V$. The optimization problem $\mathbf{P}$ can be formulated as nonlinear programming problem.

Given: $\kappa, d, T, V, f, R_{\max }$, and $N$

Minimize:

$$
\delta=\kappa \log (r)+\alpha(d, T, f) r \times 10^{-3}
$$

subject to:

$$
\begin{gathered}
r<R_{\max } \\
0<f<1000 \mathrm{KHz} \\
V \leq \frac{N r^{3}}{2}
\end{gathered}
$$

Constraint (10) requires the transmission distance to be within the transmission capability $R_{\max }$ of the acoustic modem. Constraint (11) ensures that frequency range is valid per Eq. (8). Constraint (12) assures that the total volume covered by $N$ nodes is greater than or equal to a predetermined volume $V$, where $\frac{\mathrm{Nr}^{3}}{2}$ is computed in section III-A.

This model can be extended to compute the optimal transmission range using the free space path loss in EM waves [12]. In this case, the objective function is:

$$
\delta=32.4+20 \log \left(f_{c}\right)+20 \log \left(R_{k}\right)
$$

where $f_{c}$ is the signal's frequency in $\mathrm{MHz}$ and $R_{k}$ is the range in $\mathrm{Km}$. Constraints (11) will be changed according to the frequency band of the EM waves. Eq. (2) can be substituted with the SNR equation in EM wave as follows:

$$
S N R=P_{R} / P_{N}
$$

where $P_{R}$ is the received signal power and $P_{N}$ is the noise power in Gaussian distribution.

\section{PERFormance Evaluation}

This section presents the results of the mathematical model developed to obtain the optimal inter-node distances. First, we solve the optimization problem $\mathbf{P}$ and obtain the transmission ranges that minimize transmission loss. Then, we compute the largest possible volumes for a given number of nodes $N$ that retains a transmission loss threshold $\delta_{t h}$ which can be found from Eq. 2 as follows: 


$$
\delta_{t h}=S L-S N R_{t h}-N L
$$

The transmission loss threshold is essential to maintain certain SNR values which is necessary to compute the number of nodes in a given volume.

\section{A. System Setup}

The parameters used in our model are shown in Table I. These values are obtained from the specifications of the commercial underwater acoustic modem HAM.NODE [15]. In this model, we divide the area into four levels with depth of $2500 \mathrm{~m}$ each.

\begin{tabular}{l|c}
\hline Parameter & Values \\
\hline$\kappa$ & 15 \\
Noise bandwidth $\left(B_{n}\right)$ & $1 \mathrm{KHz}$ \\
Wind speed(w) & $0 \mathrm{~m} / \mathrm{s}$ \\
Shipping activity factor (s) & 0.5 \\
Water Acidity (pH) & 8 \\
Water salinity (S) & $35 \mathrm{ppt}$ \\
Date rate (R) & $3.4 \mathrm{Kpbs}$ \\
Temperature (T) & $15^{\circ} \mathrm{C}$ \\
$R_{M a x}$ & $30 \mathrm{~km}$ \\
BER & $10^{-9}$ \\
$V_{t h}$ & $10000 \times 10000 \times 10000$ \\
$N$ & 25 \\
$P_{t}$ & $100 \mathrm{Watts}$ \\
$d$ & $0,2500,5000,7500 \mathrm{~m}$ \\
\hline \multicolumn{2}{c}{ TABLE I }
\end{tabular}

We use Orthogonal Frequency Division Multiplexing (OFDM) as an encoding technique [2]. The amplitude and the phase of the sub-carrier are calculated using the Quadrature Amplitude Modulation (QAM) scheme [16]. Assuming we consider 16-QAM modulation with OFDM transmission in our model, BER can be computed as follows:

$$
P_{b}^{16 Q A M}=\frac{3}{2 k} \operatorname{erfc}\left(\sqrt{\frac{k}{10} \frac{E_{b}}{N_{0}}}\right)
$$

where $k$ is $\log _{2} 16$ and $E_{b} / N_{0}$ is the energy per-bit-to-noise power spectral density ratio. $E_{b} / N_{0}$ is calculated as follows:

$$
E_{b} / N_{0}=S N R \frac{B_{N}}{R}
$$

where $B_{N}$ is the noise bandwidth in $\mathrm{Hz}, R$ is the data rate in bps and SNR is $10^{S N R(d, f) / 10}$. Using Eqs. (16), (17), and parameter values given in Table I, we compute SNR values for BER values of $10^{-1}, 10^{-3}, 10^{-6}$, and $10^{-9}$. The SNR values are shown in Table II.

\section{B. System Evaluation}

The absorption coefficient $\alpha$ is calculated using Ainslie and McColm model due its simplicity and accuracy. It holds for frequencies in the range of $0<f<1000 \mathrm{KHz}$. Figure 2 shows absorption coefficient at different depths. The absorption coefficient rapidly increases with frequency and thus limits the use of larger frequencies for acoustic links

\begin{tabular}{c|c}
\hline BER & SNR \\
\hline $10^{-1}$ & 4.8919 \\
$10^{-3}$ & 13.532 \\
$10^{-6}$ & 17.4120 \\
$10^{-9}$ & 19.4711 \\
\hline \multicolumn{2}{c}{ TABLE II } \\
BER VS. SNR
\end{tabular}

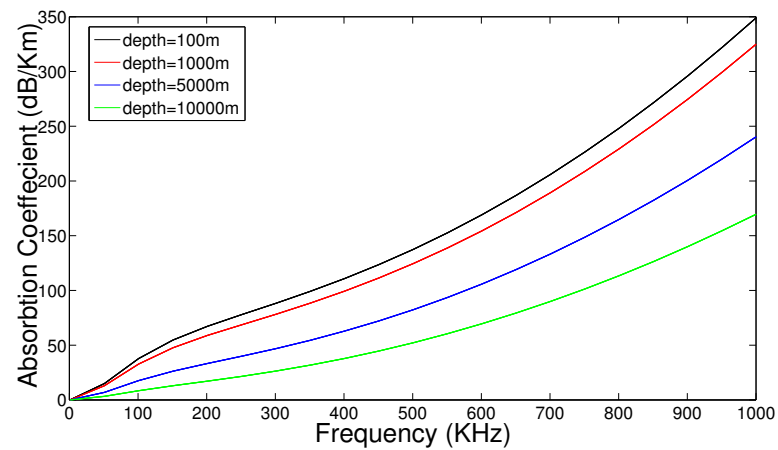

Fig. 2. Absorption coefficient $\alpha$ using Ainslie and Mccolm model at different depths for $T=15^{\circ} \mathrm{C}, p H=8$ and, $S=35$

at given distance. It is also clear that absorption coefficient decreases as depth increases.

Consequently, as the operating frequency increases, the absorption loss affects the transmission loss. Figures 3(a) and 3 (b) show the transmission loss at the depths of 10, 5000, and $10000 \mathrm{~m}$ for up to transmission range of $10000 \mathrm{~m}$ for different frequencies. Transmission loss steeply increases as transmission range increases. However, the impact of the distance is limited in low frequencies because of the low absorption loss. It is also notable that transmission loss decreases as the depth increase because of the low absorption loss in deep water.

We solved the nonlinear programming problem $\mathbf{P}$ using Optimization Toolbox in MATLAB [17]. The function fmincon is used with Active Set algorithm to find the feasible solution of the problem. Transmission loss threshold in Eq. (15) can be calculated using parameters values in Table I. Table III shows threshold values for frequencies $1,10,100$, and $1000 \mathrm{KHz}$ at depths $10,2500,5000,7500 \mathrm{~m}$ in $\mathrm{dB} / \mathrm{km}$.

\begin{tabular}{l|c|c|c|c}
\hline Depth & $10 \mathrm{KHz}$ & $100 \mathrm{KHz}$ & $500 \mathrm{KHz}$ & $1000 \mathrm{KHz}$ \\
\hline $10 \mathrm{~m}$ & 134.93 & 139.15 & 125.3 & 119.28 \\
$2500 \mathrm{~m}$ & 107.94 & 112.16 & 98.32 & 92.3 \\
$5000 \mathrm{~m}$ & 104.93 & 109.15 & 95.3 & 89.2 \\
$7500 \mathrm{~m}$ & 103.17 & 107.39 & 93.54 & 87.52 \\
\hline
\end{tabular}

TABLE III

TRANSMISSION LOSS THRESHOLD FOR $1 \mathrm{KHz}, 10 \mathrm{KHz}, 100 \mathrm{KHZ}$, AND $1000 \mathrm{KHZ}$ AT DEPTHS $10,2500,5000,7500 \mathrm{M}$ FOR A BER OF $10^{-9}$

Since the objective function has a logarithmic behavior as shown in Figure 3, the solver will always obtain the lower bound value determined by Eq. (12). In other words, the optimal transmission range $r$ is the one which solves 


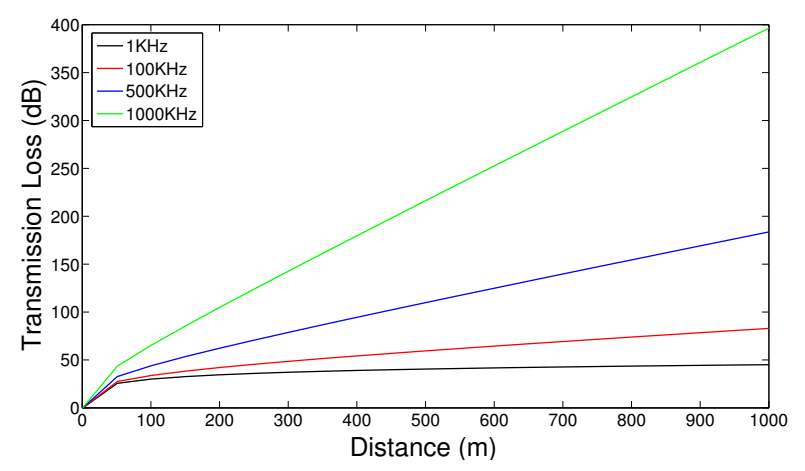

(a) Transmission loss of shallow water at depth $10 \mathrm{~m}$

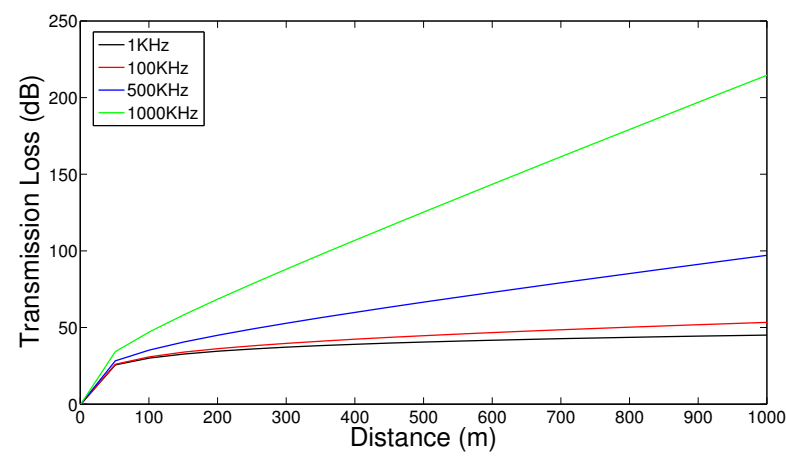

(b) Transmission loss of deep water at depth $10000 \mathrm{~m}$

Fig. 3. Transmission loss of deep and shallow water

the equation. The minimum number of nodes that gives the highest volume at each depth with minimum total transmission loss is computed. The minimum number of nodes and the corresponding transmission ranges are given in Table IV and $\mathrm{V}$, respectively. The number of nodes increases with the frequency because of the rapid increase in transmission loss, and hence the inter-node distance decreases. As the depth increases, less number of nodes are required, thus increasing the inter-node distance for the same frequency.

\begin{tabular}{l|c|c|c}
\hline Depth & $100 \mathrm{KHz}$ & $500 \mathrm{KHz}$ & $1000 \mathrm{KHz}$ \\
\hline $0-2500 \mathrm{~m}$ & 40 & 2282 & 35855 \\
$2500-5000 \mathrm{~m}$ & 37 & 3158 & 62715 \\
$5000-7500 \mathrm{~m}$ & 15 & 1853 & 44211 \\
$7500-10000 \mathrm{~m}$ & 6 & 1082 & 29969 \\
\hline
\end{tabular}

TABLE IV

MINIMUM NUMBER OF NODES AT DIFFERENT DEPTHS

\begin{tabular}{l|c|c|c}
\hline Depth & $100 \mathrm{KHz}$ & $500 \mathrm{KHz}$ & $1000 \mathrm{KHz}$ \\
\hline $0-2500 \mathrm{~m}$ & $2320.8 \mathrm{~m}$ & $602 \mathrm{~m}$ & $239.32 \mathrm{~m}$ \\
$2500-5000 \mathrm{~m}$ & $2381.9 \mathrm{~m}$ & $541.27 \mathrm{~m}$ & $199.77 \mathrm{~m}$ \\
$5000-7500 \mathrm{~m}$ & $3218.3 \mathrm{~m}$ & $646.2 \mathrm{~m}$ & $224.46 \mathrm{~m}$ \\
$7500-10000 \mathrm{~m}$ & $4367.9 \mathrm{~m}$ & $773.12 \mathrm{~m}$ & $255.82 \mathrm{~m}$ \\
\hline
\end{tabular}

TABLE V

MAXIMUM TRANSMISSION RANGE AT DIFFERENT DEPTHS
Alternatively, the maximum volume of certain number of nodes $N$ that holds the transmission loss constraint is computed. Using transmission loss threshold values in Table III, the corresponding transmission range from Eq. (7) is obtained.

There exists an optimal frequency that holds the transmission threshold and gives the largest transmission range at certain depth, transmission power, and BER. This is due to the behavior of the ambient noise model where the ambient noise approach a minimum value at frequencies around 40 $\mathrm{KHz}$. Figure 4 shows that the transmission range increases as the depth increases. The optimal frequency increases with depth because of the limited effect of absorption in deep water. Low BER values limit the transmission range for the same frequency at the same depth. Figures 5(a) and 5(b) show the optimal frequency with different values of the desired BER at depths 100 and $1000 \mathrm{~m}$, respectively. The figures also show that for a desired BER value ber $_{1}$, a maximum transmission range $r_{1}$ can be obtained. For any ber $r_{2}<b e r_{1}$, the transmission range $r_{2}$ should be less than $r_{1}$ to compensate the SNR threshold value. Increasing the transmission power level improves SNR and thus increases the inter-node distance as shown in Figures 6(a) and 6(b). Modulation scheme is an important factor that has an impact on SNR in underwater communication channels. Binary Phase-Shift Keying (BPSK) and Quadrature Phase-Shifting Keying (QPSK) are modulation schemes that use Phase-Shift Keying technique to convey data with 1 bit per symbol and 2 bits per symbol, respectively. BER of BPSK and QPSK can be computed as follows:

$$
P_{b}^{P B S K, Q P S K}=\frac{1}{2} \operatorname{erfc}\left(\frac{E_{b}}{N_{0}}\right)
$$

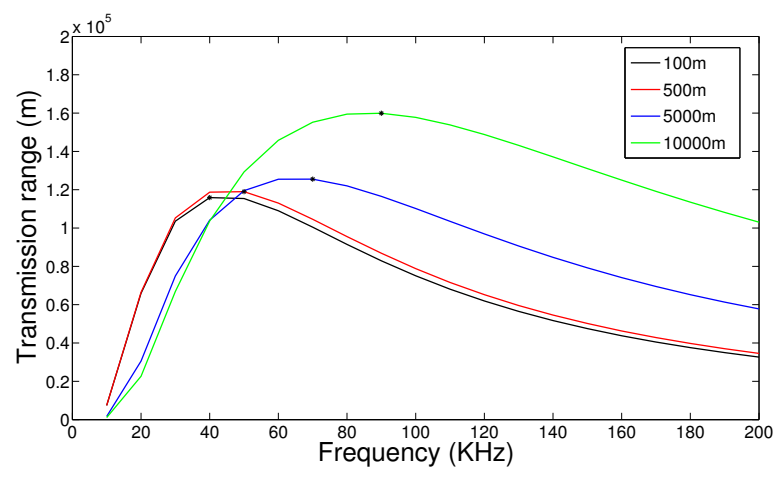

Fig. 4. Maximum transmission range for different depths with $\mathrm{P}=100 \mathrm{~W}$ and $\mathrm{BER}=10^{-9}$. The $*$ represents the optimal value

Figures 7(a) and 7(b) demonstrate the effect of modulation scheme on the inter-node distance at the optimal frequency at depths of 100 and $10000 \mathrm{~m}$, respectively. BPSK and QPSK provide larger transmission ranges because they use fewer bits of modulation compare to 8-QAM and 16-QAM. Low data rate networks, i.e., UWSN, are more susceptible to transmission loss and hence using lower bits per symbol improves the acoustic channel throughput. 


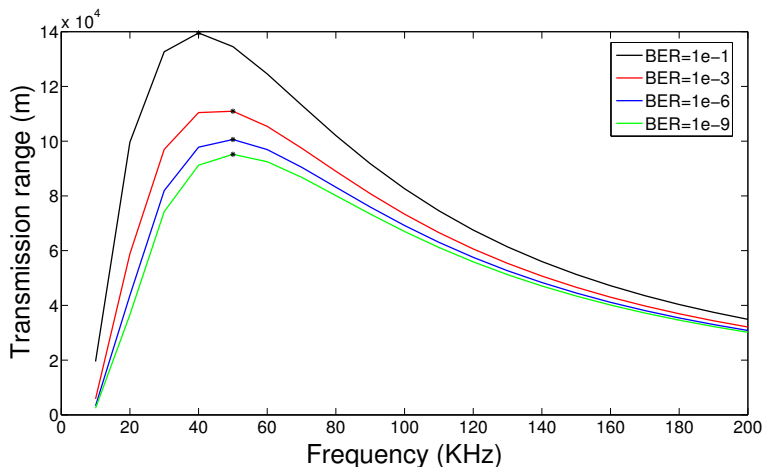

(a) Maximum transmission range at depth $100 \mathrm{~m}$ and transmission power of $100 \mathrm{~W}$ varies with the desired BER

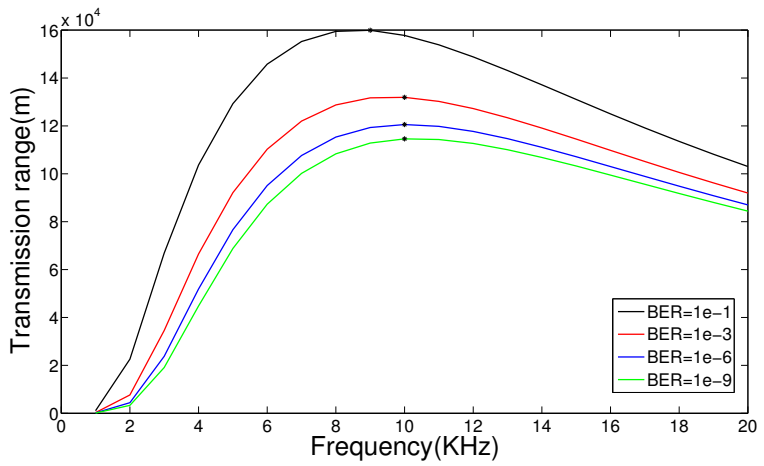

(b) Maximum transmission range at depth $10000 \mathrm{~m}$ and transmission power of $100 \mathrm{~W}$ varies with the desired BER

Fig. 5. Optimal frequency with different BER values. The $*$ represents the optimal value

\section{Nodes Placement}

Once $r$ is obtained, the coordinates of the node in 3D space are computed using Eq. (1). The nodes are placed next to each other in the 3D space forming tiled TOs that cover certain volume.

\section{Simulation}

We validate the results obtained from the mathematical model using ns-3 simulator [18]. ns-3 supports underwater acoustic networks using the available UAN framework. The framework consists of three main components: medium channel, physical (PHY), and MAC. We modified the framework as follows: the modified medium channel supports different absorption models, i.e., Fisher and Simmons, Ainslie and McColm, and Thorp models. We use Ainslie and McColm model in the simulation to assure consistency with the model. Moreover, we modified the PHY layer such that it uses passive sonar equation to calculate SNR threshold as in Eq. (15). We use the provided ALOHA MAC protocol in the framework. However, it has been adjusted to work with UDP socket in ns-3 in order to work with UDP client and server application. 1

\footnotetext{
${ }^{1}$ We intend to make our modified software packages available to the research community
}

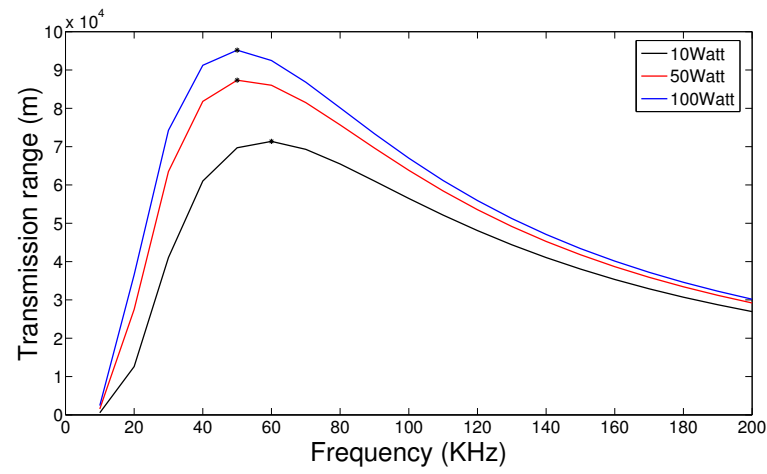

(a) Maximum transmission range at depth $100 \mathrm{~m}$ and BER of $10^{-9}$ with different transmit power

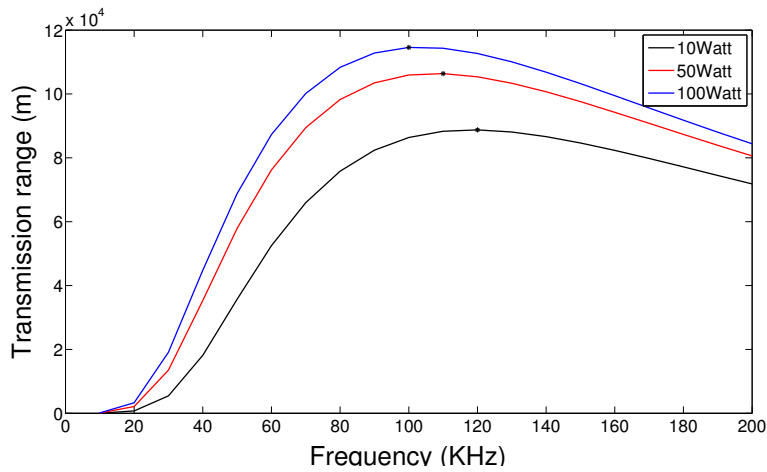

(b) Maximum transmission range at depth $10000 \mathrm{~m}$ and BER of $10^{-9}$ with different transmit power

Fig. 6. Optimal frequency with different transmission power level. The $*$ represents the optimal value

The parameters used in the simulation are listed in Table I. A node sends a dummy packet of size 25 bytes at data rate of $2 \mathrm{Kbps}$. We targeted two nodes with a distance of $10 \mathrm{~m}$ from each other as shown in Figure 8. The right node is moving away at constant velocity of 10 meters per minute. Left node continuously sends UDP packets to the right node. The packet is correctly received with probability greater than $90 \%$ if the SNR is greater than the cut-off threshold. This threshold is calculated using Eq. (17) to maintain a channel BER of $1^{-9}$. Figures 9, 10, 11, and 12 show the Packet Error Rate (PER) over the distance as node is moving away at different depths and frequencies. PER drops to 0 after certain transmission range because the SNR values degraded below the cut-off threshold value. The dashed vertical lines show the maximum transmission ranges obtained from the mathematical model in Table VI.

\section{CONCLUSIONS}

In this paper we presented an optimal node placement strategy for underwater wireless sensor networks that considers the characteristics of underwater acoustic channels. We formulated the problem as a nonlinear programming model. The objective is to obtain the transmission range that minimizes the transmission loss for a given frequency at certain depth. 


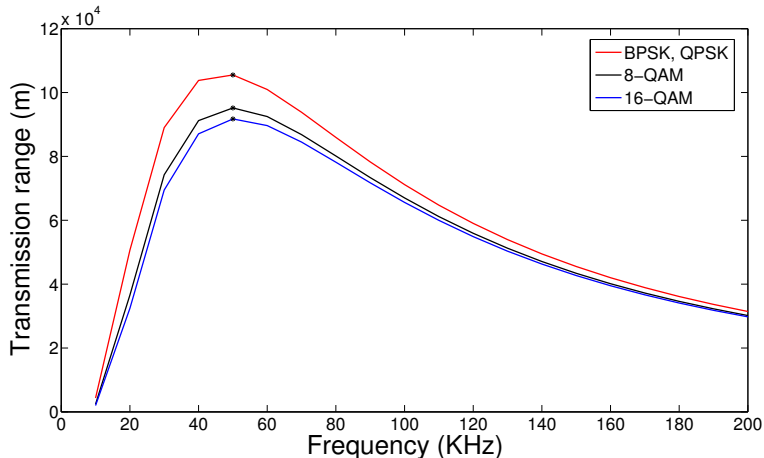

(a) Maximum transmission range for various modulation schemes at depth $100 \mathrm{~m}, 100 \mathrm{~W}$, and BER of $10^{-9}$

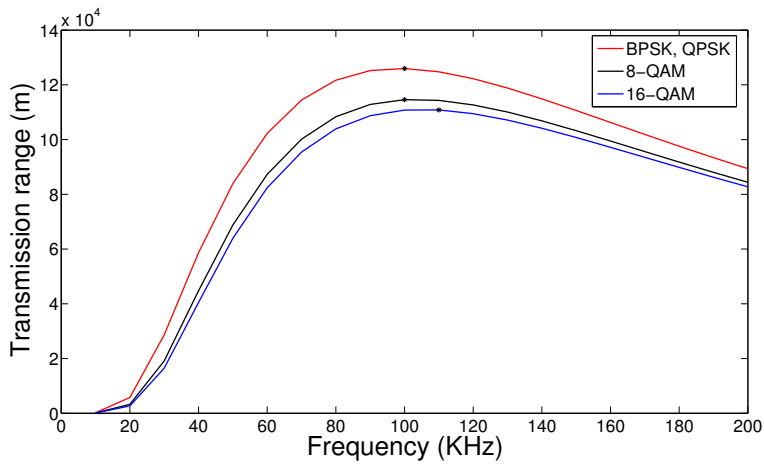

(b) Maximum transmission range for various modulation schemes at depth $10000 \mathrm{~m}, 100 \mathrm{~W}$, and BER of $10^{-9}$

Fig. 7. Optimal frequency with different modulation schemes. The * represents the optimal value

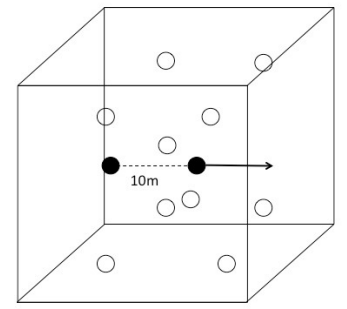

Fig. 8. Two nodes distanced $10 \mathrm{~m}$ from each other. The dashed arrow shows movement of the node

\begin{tabular}{l|c|c|c}
\hline Depth & $100 \mathrm{KHz}$ & $500 \mathrm{KHz}$ & $1000 \mathrm{KHz}$ \\
\hline $10 \mathrm{~m}$ & $2334.5 \mathrm{~m}$ & $602.93 \mathrm{~m}$ & $237.95 \mathrm{~m}$ \\
$2500 \mathrm{~m}$ & $2393 \mathrm{~m}$ & $541.28 \mathrm{~m}$ & $199.46 \mathrm{~m}$ \\
$5000 \mathrm{~m}$ & $3232.3 \mathrm{~m}$ & $646.23 \mathrm{~m}$ & $235.63 \mathrm{~m}$ \\
$7500 \mathrm{~m}$ & $4394.7 \mathrm{~m}$ & $773.16 \mathrm{~m}$ & $255.52 \mathrm{~m}$
\end{tabular}

TABLE VI

TRANSMISSION RANGE CORRESPONDING TO TRANSMISSION LOSS THRESHOLD

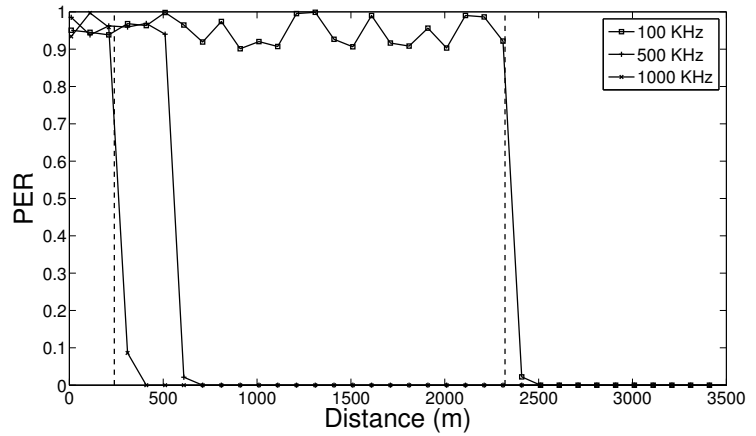

Fig. 9. Maximum transmission range to maintain a cut-off SNR threshold of $19.47 \mathrm{~dB}$ at depth of $10 \mathrm{~m}$ with frequencies 100,500 and $1000 \mathrm{KHz}$

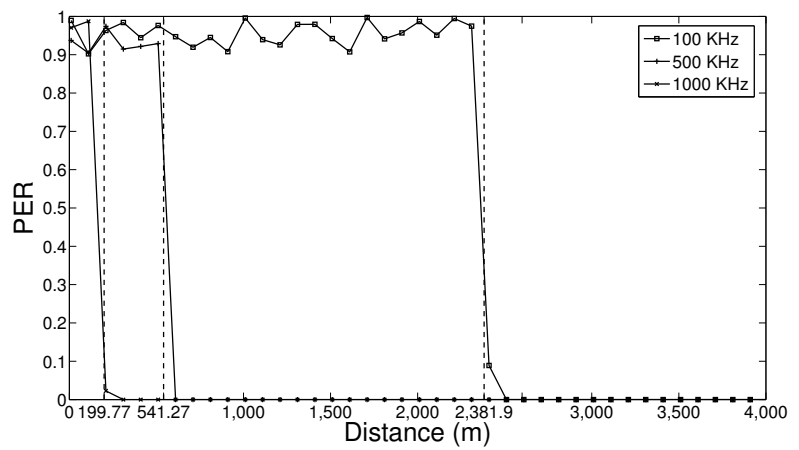

Fig. 10. Maximum transmission range to maintain a cut-off SNR threshold of $19.47 \mathrm{~dB}$ at depth of $2500 \mathrm{~m}$ with frequencies 100,500 , and $1000 \mathrm{KHz}$

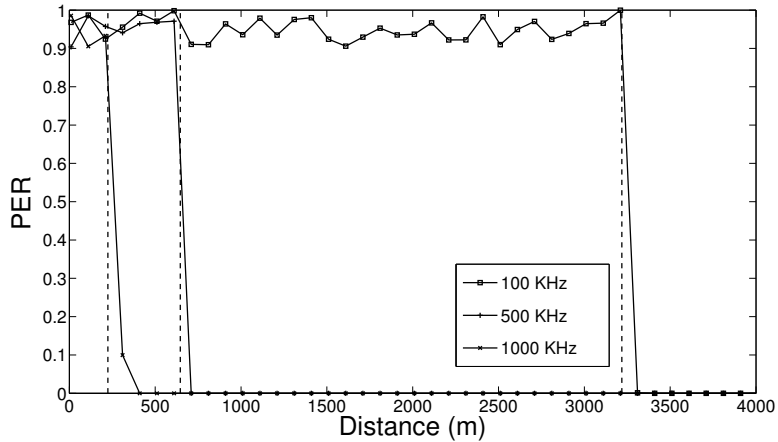

Fig. 11. Maximum transmission range to maintain a cut-off SNR threshold of $19.47 \mathrm{~dB}$ at depth of $5000 \mathrm{~m}$ with frequencies 100,500 , and $1000 \mathrm{KHz}$

We also considered two objective factors which are finding the largest volume for a given number of nodes and finding the minimum number of nodes to cover a certain volume. We computed transmission loss threshold by varying the values of BER and transmission power levels. We found that there exists an optimal frequency which gives the maximum transmission range. This optimal frequency is around $40 \mathrm{KHz}$ for different values of BER and transmission power levels.

Results showed that the operating frequency affects the 


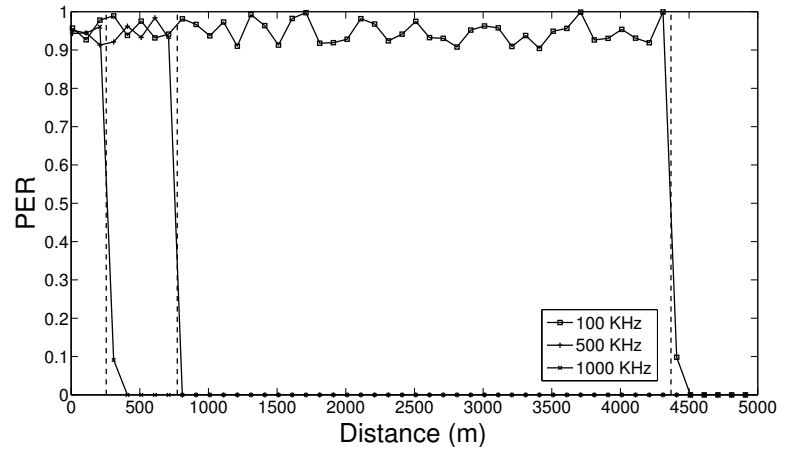

Fig. 12. Maximum transmission range to maintain a cut-off SNR threshold of $19.47 \mathrm{~dB}$ at depth of $7500 \mathrm{~m}$ with frequencies 100,500 , and $1000 \mathrm{KHz}$

number of nodes required to cover a definite volume. As frequency increases, more nodes are required with short internode distance to maintain a transmission loss threshold. Internode distance is expanded as the transmission power increases at the same water depth. We also observed that the modulation schemes have an important impact on the inter-node distance for the BER. It is shown that schemes that use lower bit per symbols increase channel throughput as well as transmission range. We validated the results obtained from the mathematical model using ns-3.

For future work, we aim to consider extending the objective function to include the channel capacity. Channel capacity is an important factor that improves the overall performance of the underwater network. This model can be used as an initial infrastructure for future researches in UWSN.

\section{REFERENCES}

[1] M. Stojanovic, "On the relationship between capacity and distance in an underwater acoustic communication channel," ACM SIGMOBILE Mobile Computing and Communications Review, vol. 11, no. 4, pp. 34-43, 2007.

[2] I. Akyildiz, D. Pompili, and T. Melodia, "Underwater acoustic sensor networks: research challenges," Ad hoc networks, vol. 3, no. 3, pp. 257279, 2005.

[3] K. Akkaya and A. Newell, "Self-deployment of sensors for maximized coverage in underwater acoustic sensor networks," Computer Communications, vol. 32, no. 7-10, pp. 1233-1244, 2009.

[4] D. Pompili, T. Melodia, and I. Akyildiz, "Deployment analysis in underwater acoustic wireless sensor networks," in Proceedings of the 1st ACM international workshop on Underwater networks. ACM, 2006, pp. $48-55$.

[5] S. Alam and Z. Haas, "Coverage and connectivity in three-dimensional underwater sensor networks," Wireless Communications and Mobile Computing, vol. 8, no. 8, pp. 995-1009, 2008.

[6] R. Urick, "Principles of underwater sound," New York, 1983

[7] R. Coates, Underwater acoustic systems. Halsted Pr, 1989.

[8] I. Akyildiz, D. Pompili, and T. Melodia, "State-of-the-art in protocol research for underwater acoustic sensor networks," in Proceedings of the 1st ACM international workshop on Underwater networks. ACM, 2006, pp. 7-16.

[9] S. Ibrahim, J. Cui, and R. Ammar, "Efficient surface gateway deployment for underwater sensor networks," in Computers and Communications, 2008. ISCC 2008. IEEE Symposium on. IEEE, 2008, pp. 1177-1182.

[10] M. Domingo, "Optimal placement of wireless nodes in underwater wireless sensor networks with shadow zones," in Wireless Days (WD), 2009 2nd IFIP. IEEE, 2009, pp. 1-6.

[11] M. Ainslie and J. McColm, "A simplified formula for viscous and chemical absorption in sea water," Journal of the Acoustical Society of America, vol. 103, no. 3, pp. 1671-1672, 1998.
[12] J. Walfisch and H.L. Bertoni, "A theoretical model of UHF propagation in urban environments," Antennas and Propagation, IEEE Transactions on, vol. 36, no. 12, pp. 1788-1796, 1988.

[13] C. van Moll, M. Ainslie, and R. van Vossen, "A simple and accurate formula for the absorption of sound in seawater," Oceanic Engineering, IEEE Journal of, vol. 34, no. 4, pp. 610-616, 2009.

[14] S. Ibrahim, R. Ammar, and J. Cui, "Surface gateway placement strategy for maximizing underwater sensor network lifetime," Computers and Communications (ISCC), 2010 IEEE Symposium on. IEEE, 2010, pp. $342-346$.

[15] "Develogic Subsea Systems," http://www.develogic.de/.

[16] J. Proakis, "Digital communications. 1995."

[17] "Mathworks," http://www.mathworks.com/help/toolbox/optim/.

[18] "ns-3," http://www.nsnam.org/. 